\title{
A musicoterapia em oncologia: percepções de crianças e adolescentes em cuidados paliativos
}

\author{
Music therapy in oncology: perceptions of children and adolescents in palliative care \\ Musicoterapia en oncología: percepciones de niños y adolescentes en cuidados paliativos
}

Julia Helena Machado Franco ${ }^{1}$ (C) Carla Braz Evangelista ${ }^{1,2}$ (]) Mariana de Sousa Dantas Rodrigues ${ }^{1,2}$ (1) Ronny Anderson de Oliveira Cruz ${ }^{1,2}$ (1) Indaya da Silva Machado Freire Franco ${ }^{3}$ (1) Mayara Limeira Freire ${ }^{3,4}$ (1)

1. Centro Universitário de João Pessoa. João Pessoa, Paraíba, Brasil.

2. Universidade Federal da Paraíba, Programa de Pós-Graduação em Enfermagem. João Pessoa, Paraíba, Brasil.

3. Universidade Federal da Paraíba. João Pessoa, Paraíba, Brasil.

4. Faculdade Brasileira de Ensino, Pesquisa e Extensão. João Pessoa, Paraíba, Brasil.
Autor correspondente:

Carla Braz Evangelista.

E-mail: carlabrazevangelista@gmail.com.

Recebido em 01/02/2021.

Aprovado em 02/06/2021.

DOl:https://doi.org/10.1590/2177-9465-EAN-2021-0012

\section{Resumo}

Objetivo: analisar as percepções de crianças e adolescentes com câncer em cuidados paliativos sobre a musicoterapia. Método: pesquisa de campo, com abordagem qualitativa, realizada com sete crianças e adolescentes hospitalizadas. A técnica de desenho-estória foi realizada inicialmente a partir do desenho sobre os sentimentos diante da hospitalização e a estória do desenho. Em seguida, houve a interação musical, desenho sobre o significado da música diante do processo de hospitalização, e a estória do desenho. Para análise, utilizou-se a técnica de análise de conteúdo. Resultados: antes da musicoterapia, crianças e adolescentes expressaram sentimentos de tristeza, medo e saudade relacionados ao rompimento do vínculo familiar durante o processo de hospitalização. Após receberem a musicoterapia, elas expressaram as sensações de prazer e bem-estar que essa vivência pode proporcionar, permitindo-Ihes a expressão dos sentimentos mais profundos. Conclusão: a musicoterapia pode beneficiar a criança e o adolescente com câncer uma vez que permite a expressão de sentimentos, possibilita o resgate de lembranças e proporciona esperança diante da situação vivenciada, além do alívio da dor. Implicações para a prática: a musicoterapia contribui no processo paliativista, podendo conferir uma melhor qualidade de vida às crianças e adolescentes com câncer

Palavras-chave: Musicoterapia; Cuidados Paliativos; Câncer; Criança; Adolescente.

\section{Abstract}

Objective: to analyze the perceptions of children and adolescents with cancer in palliative care about music therapy. Method: field research with a qualitative approach, conducted with seven children and adolescents. The drawing-story technique was initially carried out based on drawings on feelings about hospitalization and the drawing story. Then there was the musical interaction, drawing on the meaning of music in the face of the hospitalization process, and the story of the drawing. For analysis, the content analysis technique was used. Results: before music therapy, the children and adolescents expressed feelings of sadness, fear and longing related to the breaking of the family bond during the hospitalization process. After receiving music therapy, they expressed feelings of pleasure and well-being that this experience can provide them, allowing them to express their deepest feelings. Conclusion: music therapy can benefit children and adolescents with cancer as it allows for the expression of feelings, enables the rescue of memories and provides hope in the face of the situation experienced, in addition to pain relief. Implications for the practice: music therapy contributes to the palliative process and can offer better quality of life to children and adolescents with cancer.

Keywords: Music Therapy; Palliative Care; Cancer; Child; Adolescent

\section{Resumen}

Objetivo: analizar las percepciones de niños y adolescentes con cáncer en cuidados paliativos sobre la musicoterapia. Método: investigación de campo con enfoque cualitativo, realizada con siete niños y adolescentes. La técnica del dibujo-cuento se llevó a cabo inicialmente basándose en el dibujo de los sentimientos sobre la hospitalización y en el cuento del dibujo. Luego hubo interacción musical, con dibujo sobre el significado de la música durante el proceso de hospitalización y el cuento del dibujo. Para el análisis, se utilizó la técnica de análisis de contenido. Resultados: antes de la musicoterapia, los niños y adolescentes expresaron sentimientos de tristeza, miedo y anhelo relacionados con la ruptura del vínculo familiar durante el proceso de hospitalización. Después de recibir musicoterapia, manifestaron las sensaciones de placer y bienestar que esta experiencia les puede brindar, permitiéndoles expresar sus sentimientos más profundos. Conclusión: la musicoterapia puede beneficiar a niños y adolescentes con cáncer ya que permite la expresión de sentimientos, posibilita el rescate de recuerdos es fuente de esperanza ante la situación vivida, además de aliviar el dolor. Implicaciones para la práctica: la musicoterapia contribuye a proceso paliativo y puede brindar una mejor calidad de vida a los niños y adolescentes con cáncer.

Palabras clave: Musicoterapia; Cuidados Paliativos; Cáncer; Niño; Adolescente. 


\section{INTRODUÇÃO}

O cuidado paliativo é uma abordagem que auxilia na melhoria da qualidade de vida de pacientes e familiares que se encontram com doenças que ameaçam a vida, mediante prevenção, investigação e tratamento da dor e de outros sintomas psicológicos, sociais e espirituais ${ }^{1}$.

Na pediatria, os cuidados paliativos se tornam um campo específico, sendo fornecidos em qualquer nível de assistência, exigindo da equipe multidisciplinar uma avaliação ampla que contemple as dimensões físicas, psíquicas, sociais e espirituais. Iniciam no momento em que ocorre o diagnóstico e continuam durante todo o processo de adoecimento, independente da realização de terapia convencional ou não, sendo adaptados aos valores do paciente e da família, a qual também é incluída no atendimento. Estes são uma realidade importante na área da saúde e de grande relevância na assistência a crianças e adolescentes ${ }^{2}$.

Ressalta-se que nem todas as crianças e adolescentes podem ser beneficiadas pelo tratamento curativo oncológico, porém o alívio do sofrimento proporcionado pelos cuidados paliativos pode ser alcançado ${ }^{3}$. Essa abordagem torna-se indispensável, principalmente diante da impossibilidade de cura, pelo fato do diagnóstico de câncer ser uma situação geradora de muito estresse e por envolver um tratamento doloroso $\mathrm{S}^{4}$.

Pesquisas vêm verificando os benefícios de intervenções não farmacológicas aos pacientes com câncer e/ou cuidados paliativos, com destaque para as crianças e adolescentes ${ }^{5-9}$. Estudo de revisão teve o objetivo de identificar as intervenções de enfermagem utilizadas nos cuidados paliativos voltadas para a criança e o adolescente com câncer e mostrou que a massagem, a musicoterapia, os exercícios físicos e a utilização do brinquedo terapêutico são terapias de grande relevância nos cuidados paliativos, principalmente quando direcionadas a um sintoma específico ${ }^{5}$.

Estudo com objetivo de identificar intervenções não farmacológicas para fadiga em crianças e adolescentes com câncer, evidenciou resultados positivos para a realização de exercícios, exercícios acompanhados de atividades de lazer, toque terapêutico e acupuntura. ${ }^{6}$ Evidências científicas sobre terapias não farmacológicas para manejo da fadiga e estresse psicológico em crianças e adolescentes com câncer também verificou o benefício de intervenções diante desses problemas ${ }^{7}$.

Estudo de revisão sistemática avaliou o uso das terapias complementares no tratamento da dor em pacientes oncológicos que se encontravam em cuidados paliativos e verificou os benefícios da massagem terapêutica, relaxamento muscular progressivo e imagem guiada. Entretanto, inferiu a necessidade de novas pesquisas para avaliar a eficácia da acupuntura ${ }^{10}$. Por sua vez, pesquisa avaliou a associação entre a intervenção de palhaço e os níveis de estresse psicológico e fadiga em crianças e adolescentes com câncer e verificou que esta terapia pode ser benéfica para este público tendo em vista que os níveis totais de estresse e fadiga melhoraram. ${ }^{8}$ Além dessas supracitadas, outras intervenções também são consideradas: hipnose, meditação, arteterapia, yoga, acupuntura, reiki e toque terapêutico².

Evidências sobre as intervenções não farmacológicas direcionadas ao público infantojuvenil com câncer despertam o interesse pelo tema, estimulam os profissionais e destacam a importância do tratamento convencional associado a uma terapia não farmacológica para a melhoria da qualidade de vida diante da situação de saúde? .

No contexto das terapias não farmacológicas, destaca-se a musicoterapia, uma vez que a realização desta intervenção poderá permitir o alívio de manifestações como ansiedade, dor e fadiga e efeitos secundários do tratamento oncológico, consequentemente melhorando a qualidade de vida de pacientes com câncer. Também pode reduzir a necessidade de drogas anestésicas, analgésicas e o tempo de recuperação e de internação ${ }^{11}$.

Neste contexto, é importante considerar que as terapias não farmacológicas podem auxiliar a criança e o adolescente com câncer na diminuição dos sintomas físicos, psicológicos, sociais e espirituais, e que a música pode ser uma das terapias utilizadas para aliviar o sofrimento, a dor e a tensão desta, permitindo, também, um momento de descontração e lazer. Assim, esta pesquisa tem como objetivo analisar as percepções de crianças e adolescentes com câncer em cuidados paliativos sobre a musicoterapia.

\section{MÉTODO}

Trata-se de uma pesquisa de campo, com abordagem qualitativa, realizada no setor de Pediatria de um hospital localizado no município de João Pessoa/PB. A pesquisa seguiu recomendações do Consolidated criteria for reporting qualitative research (COREQ) permitindo a melhora do rigor da pesquisa ${ }^{12}$.

A escolha da referida instituição deu-se em decorrência de ser um hospital referência para o tratamento oncológico que possui diversos setores, dentre eles o ambulatório, unidade de terapia intensiva, bloco cirúrgico, urgência e pediatria; e pelo fato de a pesquisadora principal realizar um projeto voluntário no setor de Pediatria deste local, a partir das práticas de arteterapia e musicoterapia, o que facilitou o acesso ao serviço e a aproximação com os profissionais que ali atuavam.

A amostra se deu por conveniência, e foi composta dos sete participantes que atenderam aos critérios de inclusão da pesquisa: possuir idade entre oito e dezenove anos; estar internados no hospital; e estar em condições clínicas para participar das atividades de pesquisa. Foram excluídas aqueles que estavam sendo acompanhados no ambulatório, na Unidade de Terapia Intensiva ou que já participaram da musicoterapia anteriormente. Destaca-se que a pesquisa foi finalizada considerando o intervalo de tempo proposto para a coleta de dados, qualidade do material, análise de conexão entre o material empírico de cada participante, ausência de informações novas e consequentemente saturação dos dados.

Por sua vez, a escolha da faixa etária deveu-se a facilidade de compreensão das perguntas por esse público, tendo em vista 
que crianças com menor idade poderiam não compreender o que foi solicitado ou até mesmo não conseguir expor seus sentimentos.

A coleta de dados com a população em uma faixa etária superior a sete anos, permite um melhor desenvolvimento cognitivo, e consequentemente possibilita uma maior abordagem da experiência ${ }^{4}$.

Os dados foram coletados de março a abril de 2019, a partir de instrumento baseado em estudos ${ }^{13-14}$, e envolvendo um roteiro de perguntas para a técnica do desenho-estória (D-E). A técnica do D-E foi desenvolvida por Walter Trinca e constitui uma ferramenta que une o desenho livre e a contação de estórias, permitindo, desse modo, que os indivíduos revelem situações conflitantes e problemas emocionais em uma folha de papel ${ }^{15}$. Ressalta-se que houve uma adaptação à técnica $D-E e$ não foi utilizada a sequência de cinco desenhos. Antes do início da coleta de dados, a pesquisadora principal foi devidamente treinada para realização desta atividade. Para caracterização dos dados sociodemográficos também se utilizou um instrumento com dados sobre gênero, idade, raça, escolaridade e diagnóstico.

A técnica foi realizada em dois momentos: no primeiro, solicitou-se a realização de um desenho sobre o modo como cada criança e adolescente estavam se sentindo diante da hospitalização; em seguida, pediu-se que contassem uma estória do desenho realizado. Em sequência, no segundo momento, foi realizada a interação musical, mediante pré-seleção pelos próprios participantes, sendo o modo de entrega ao vivo, voz e violão. Quando não era possível devido à escolha da música, a apresentação da música foi feita por meio de aparelho de som. Para a escolha da música, a coletadora questionava sobre a música que o participante gostaria de ouvir.

Após a interação musical, a entrevista envolveu perguntas sobre o que os momentos musicais representaram para cada um. Para responder, os participantes realizaram um novo desenho, contando a estória. Ambos os momentos foram concretizados individualmente e foram gravados em aparelho MP3. Cumpre assinalar que a sequência das etapas foi realizada em um único dia, no lugar onde criança ou adolescente se encontrava, com uma média de duração de aproximadamente 60 minutos por participante.

As falas e os desenhos foram codificados por meio de cores primárias e secundárias (amarelo, azul, azul claro, roxo, rosa, verde, vermelho) escolhidas pelas crianças e adolescentes a fim de manter o anonimato e o sigilo das informações.

A análise de dados foi realizada mediante a técnica de análise de conteúdo, em conformidade com as fases de préanálise, exploração do material e tratamento dos resultados (inferência e interpretação) ${ }^{16}$.

Nesta pesquisa foram considerados os aspectos éticos pertinentes as pesquisas envolvendo seres humanos, de acordo com a Resolução $n^{\circ}$ 466/12 principalmente no que diz respeito ao Termo de Consentimento Livre e Esclarecido direcionado ao responsável pela criança e o Termo de assentimento para criança e adolescente ${ }^{17}$, sendo aprovada pelo Comitê de Ética do Centro Universitário de João Pessoa-UNIPÊ (CEP/UNIPÊ) com CAAE no 07136819.4.0000.5176.

\section{RESULTADOS}

Participaram do estudo sete crianças e adolescentes com idade variando de oito a dezessete anos, das quais três (43\%) eram do eram do sexo feminino e quatro (57\%) eram do sexo masculino. A maioria era parda, com seis (86\%), e apenas uma (14\%) era branca. Todas as crianças eram escolarizadas, e a maioria apresentava Ensino Fundamental I completo, com quatro (57\%), seguido das que se encontravam no Ensino Fundamental II, com uma (14\%), e apenas duas (29\%) encontravam-se no Ensino Médio. Três (43\%) dos participantes apresentavam leucemia e os demais apresentavam linfoma, sarcoma de membros inferiores, neoplasia maligna renal e câncer na tireóide (14,3\%).

No que diz respeito aos dados da pesquisa, a análise do material empírico permitiu a construção de duas categorias que serão apresentadas a seguir:

\section{CATEGORIA I - Compreensão sobre o processo de hospitalização}

Nesta categoria, as crianças e adolescentes compreenderam o processo de hospitalização a partir de sentimentos negativos, conforme apresentado na Figura 1 e relatos abaixo.

Em relação ao sentimento de tristeza por estar hospitalizado, pode-se observar nos desenhos e principalmente nas falas dos depoentes Verde e Roxo. $\mathrm{O}$ depoente Verde mostra várias nuvens e a presença da chuva, significando a lágrima e a tristeza de estar doente e hospitalizado, e a depoente Roxo verbaliza sobre a tristeza na descoberta da doença e no seu processo de hospitalização.

Quando fui hospitalizado pela primeira vez chorava todos os dias, hoje me sinto triste, mas não choro mais (VERDE).

Essa sou eu, e esse é o hospital. Estou do lado de fora do hospital. Já me senti muito triste por estar no hospital, mas já me acostumei (AZUL-CLARO).

É claro que não gosto de ficar aqui no hospital, por mais que eu saiba que é aqui que vou ficar melhor [...] (AMARELO).

[...]. Eu tô no hospital porque estou doente, e minha mãe tá esperando se eu vou poder ir pra casa (VERMELHO).

Esse sou eu na cama, e essa outra cama é do meu irmão; sinto muita falta dele. O hospital não é um lugar ruim, aqui têm pessoas que estão cuidando da gente, mas não é minha casa, me sinto só aqui. Essa cama tá vazia porque ele não tá aqui comigo (AZUL).

Me sinto muito só nesse hospital. Minha mãe não pode vir me acompanhar porque tenho um irmãozinho e ela tem que ficar com ele, normalmente; quem vem comigo é minha tia. Sinto falta de casa e da minha mãe (ROSA). 


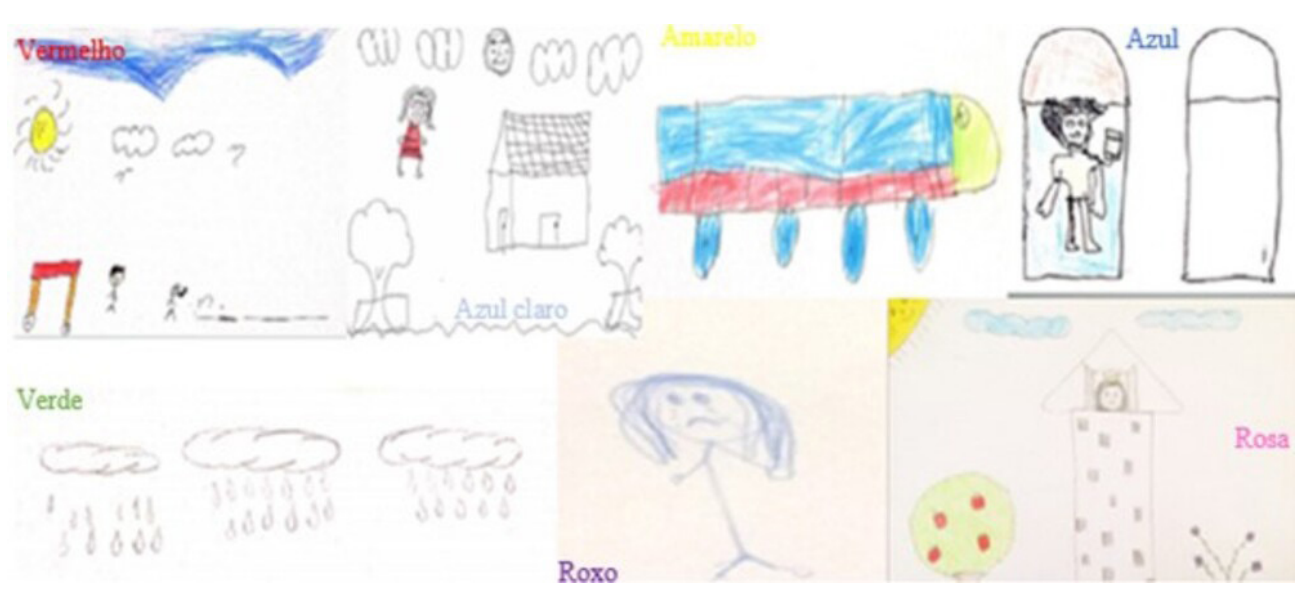

Figura 1. Desenho realizado antes da musicoterapia sobre como as crianças e adolescentes estavam se sentindo diante do processo de hospitalização. Dados da Pesquisa. João Pessoa, 2019.

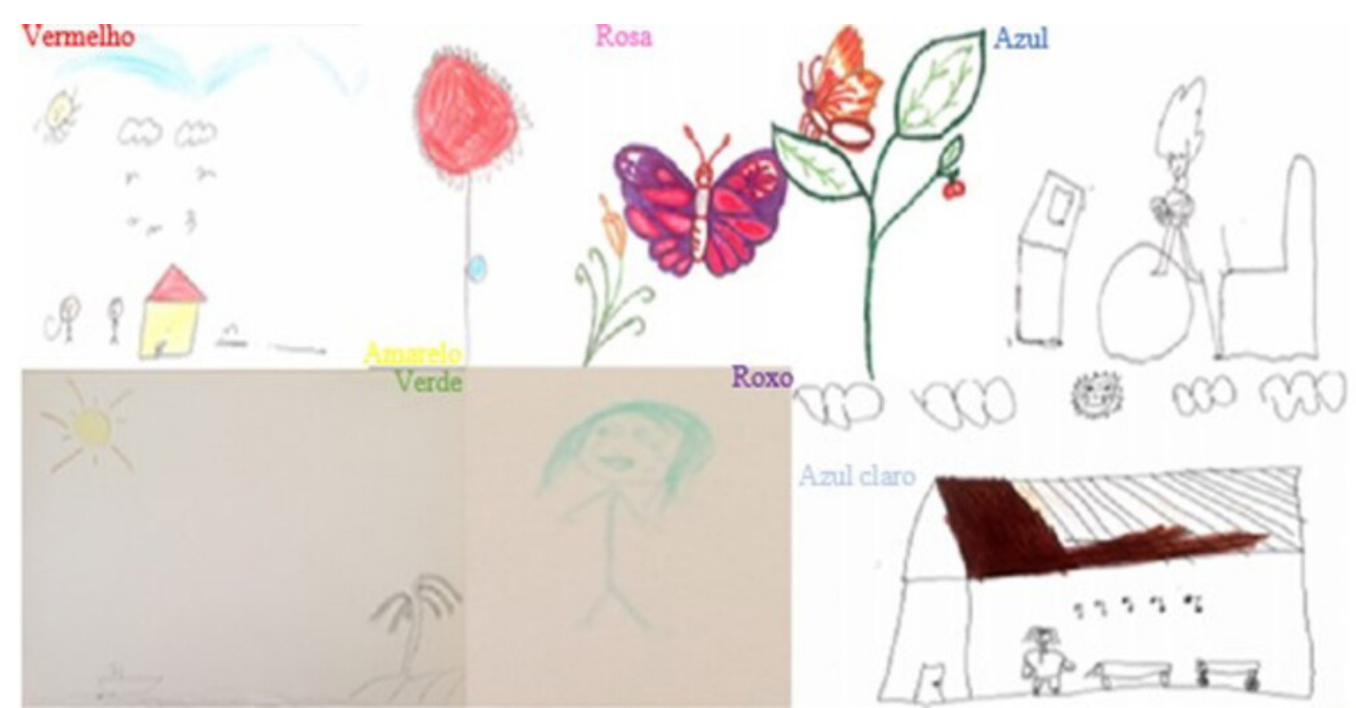

Figura 2. Desenho realizado após a musicoterapia sobre o que os momentos musicais significaram para as crianças e adolescentes. Dados da Pesquisa. João Pessoa, 2019.

Essa sou eu chorando. Quando descobri que estava doente, me senti triste e sem chão. Quando sou internada aqui no hospital é como se eu estivesse cada vez mais doente (ROXO).

Na fala da depoente Rosa, conseguimos perceber a saudade de casa e a solidão causada pela mudança do lar para um ambiente no qual a criança não possui a presença de todos os membros da família e amigos. Além disso, o seu desenho representa a princesa (depoente Rosa) em uma prisão (hospital), ou seja, representa o hospital como um ambiente solitário, no qual a criança não se sente livre. A entrevistada Azul-Claro também demonstra não querer estar no hospital. Em seu desenho, apresenta-se fora do hospital, embora já tenha se acostumado com o ambiente. Por sua vez, o depoente Amarelo verbaliza não querer estar nesse local, mas reconhece que é o único lugar que pode auxiliá-lo a melhorar sua saúde.

\section{CATEGORIA II - Percepção sobre os efeitos da musicoterapia na sua condição de saúde}

Nesta categoria, serão apresentados os efeitos da musicoterapia para as crianças e adolescentes com câncer, que se encontram em cuidados paliativos. Na percepção dos entrevistados, a música os auxilia a esquecer o que estão passando e a não desistir da vida, fazendo com que eles se lembrem de casa e de familiares, sintam-se livres e permitindo o alívio da dor, conforme apresentado na Figura 2.

Os depoimentos das crianças e adolescentes evidenciam diferentes efeitos relacionados à musicoterapia sobre a condição de saúde atual. Como é possível observar, a maioria dos participantes, ao escutar a música, lembra de suas casas. Os entrevistados Vermelho, Azul e Azul-Claro trazem essa perspectiva em seus desenhos, quando a pintura da casa se faz presente. Contudo, a percepção da entrevistada Azul-Claro está associada ao acalanto materno, realizado por meio da musicalização para o 
alívio da dor. Tem uma característica afetiva (materna/familiar) e técnica (promoção do alívio da dor). Ainda sobre o vínculo materno, o participante Amarelo o destaca como um alicerce para o enfrentamento da condição clínica e hospitalização e a partir da musicoterapia sente-se com forças para não desistir e faz lembrar da sua mãe que nunca desistiu dele.

Quando escuto essa música, fecho os olhos, sou levado para o mar. O mar, a praia é onde me sinto bem. Gosto de escutar essa música, principalmente quando estou internado, ou quando recebo uma notícia ruim sobre meu tratamento. Me faz esquecer o que estou passando (VERDE).

Escolhi essa música porque ela me ensina a não desistir, e essa flor é pra minha mãe porque ela não desistiu de mim, como meu pai fez [...] (AMARELO).

Esse sou eu e minha irmãzinha em casa, sinto muita falta dela, de brincar com ela. Escolhi essa música porque eu sempre cantava para ela. Acho que o que eu sinto mais falta é dela, e ela gostava muito, já vi vocês cantando lá fora, sempre tive vontade de participar, mas não podia sair do quarto. Fiquei feliz por esse momento hoje (VERMELHO).

Esse sou eu em casa jogando, sinto falta de casa. Gosto muito dessa música, me sinto livre quando escuto ela, livre desse hospital. Meu irmão também gosta muito dela, escutávamos juntos (AZUL).

[...] Minha mãe cantava essa música pra mim quando eu sentia muita dor. Às vezes, quando ela não tá aqui comigo fico cantando pra mim, até minha dor passar (AZUL-CLARO).

Me desenhei chorando de novo, mas essas lágrimas são diferentes [...]. Esse choro é de esperança, e essa música me ajuda e levantar e continuar meu tratamento (ROXO).

Desenhei borboletas, pois simbolizam liberdade, sinto falta de brincar, de estar livre. Quando a gente fica doente a gente não pode fazer mais nada. Essa música que eu escolhi me faz imaginar que um dia terei essa liberdade, que eu posso voar (ROSA).

Em relação à depoente Roxo, embora apresente em seu desenho lágrimas, ela relata que estas representam a esperança no restabelecimento de sua saúde com o tratamento para o câncer, o que a fortalece diante da atual situação. O mesmo pode ser observado no desenho da depoente Rosa, no qual retrata borboletas, de modo a representar uma sensação de liberdade, de voo, que não é presente durante a hospitalização. Pode-se observar nas falas das depoentes Rosa e Roxo que a música auxilia a mudar as suas percepções quanto ao hospital. Para o entrevistado Verde, a música proporciona bem-estar, mesmo diante das tristezas e notícias ruins relacionadas com o seu problema de saúde.

\section{DISCUSSÃO}

A hospitalização da criança e do adolescente pode ser considerada uma situação estressante e que interfere nas respostas comportamentais e emocionais desses sujeitos, consequentemente influencia o desenvolvimento e os resultados alcançados com a assistência prestada ${ }^{18}$.

As crianças e adolescentes internadas, em especial as com câncer, possuem uma série de sintomas físicos e psicológicos em decorrência do processo de adoecimento ${ }^{19}$. Com a internação, ocorre o rompimento das atividades cotidianas e familiares, $e$ uma nova rotina deve ser seguida, o que gera uma série de sentimentos negativos, como tristeza e ansiedade, associados aos procedimentos técnicos dolorosos (administração de medicamentos e realização de exames), as restrições hospitalares, ao isolamento, as rotinas institucionais e a saudade de amigos e do ambiente familiar ${ }^{14}$.

O diagnóstico de câncer infantojuvenil e o processo de hospitalização afetam toda a estrutura familiar e trazem diversas reações para a família. Reações de surpresa, negação, tristeza, medo, ansiedade, sofrimento, despreparo para lidar com a situação, raiva, depressão e incertezas cercam esse momento ${ }^{20}$.

Estudo com objetivo de investigar o significado do brincar para criança em isolamento hospitalar verificou que a solidão, tristeza e prisão definem o ambiente. Além disso, evidenciou-se que nesse local o paciente sofre em decorrência da doença e das restrições existentes, sentindo-se ameaçado pela presença do profissional de saúde por compreendê-lo como realizador de procedimentos ${ }^{21}$.

Embora sejam capazes de comunicar o que sentem, crianças e adolescentes podem precisar de ajuda para expressar seus sentimentos ${ }^{19}$. A oferta de apoio psicológico, práticas que melhorem o atendimento ao paciente e sua família, e que estimulam o brincar podem ter grande relevância para o bemestar do público infantojuvenil ${ }^{18}$.

Entretanto, a implementação de medidas que possam melhorar a qualidade de vida desse público por parte dos profissionais de saúde ainda é um desafio. Existe uma limitação por parte de profissionais de saúde para manejar manifestações do câncer infantojuvenil e, consequentemente, a falta de capacitação profissional para assisti-los 22 .

Neste contexto, destaca-se a técnica de desenhar, para que crianças e adolescentes expressem seus sentimentos e pensamentos ${ }^{14}$. Esta técnica pode ser implementada e diversos ambientes, sejam eles domésticos ou hospitalares, para auxiliar no diagnóstico e tratamento e permite a revelação de sensações valiosas para sua vida, sejam elas positivas ou negativas ${ }^{23}$, o que pode auxiliar os profissionais de saúde a entenderem as reais necessidades da criança e do adolescente, de modo que se possa planejar uma assistência direcionada e individualizada.

A presente pesquisa evidenciou que a musicoterapia pode ser benéfica durante o processo de hospitalização da criança e do adolescente com câncer. Sob o ponto de vista iconográfico, observou-se no presente estudo uma tendência diferenciada no conteúdo dos desenhos realizados após a intervenção musical, 
visto que foi explanado um maior quantitativo de elementos e de cores dispostos nas folhas de papel. Quanto aos personagens, é notória a conotação imagética e afetiva acerca da possibilidade de viver fora do contexto hospitalar, especialmente no ambiente doméstico, com maior proximidade dos membros familiares que possuem uma participação significativa na vida das crianças e adolescentes desta pesquisa.

A musicoterapia é considerada um recurso para a redução da dor ${ }^{24-26}$, dos níveis pressóricos, da frequência respiratória e cardíaca ${ }^{11,26}$, ansiedade ${ }^{11,24-26}$, diminuição de sintomas depressivos ${ }^{25-26}$ e do consumo de ansiolíticos ${ }^{25}$. Auxilia no desenvolvimento cognitivo, de atenção, memória e destreza e ao contrário de outras terapêuticas, a música é considerada uma modalidade de terapia eficiente, não invasiva, indolor e que apresenta poucos efeitos secundários ${ }^{24}$.

Em pacientes vulneráveis, como no caso de pacientes com câncer, a música diminui o desequilíbrio diante da hospitalização e das mudanças impostas pelo novo ambiente, rotinas, e convívio social com outros sujeitos e da perda dos vínculos com pessoas próximas podendo levá-los a restauração, em decorrência de seus efeitos evidenciados no corpo, coração, e na mente ${ }^{24}$.

Quando a música é escutada, diferente de que quando se está em atividade sem música, os indivíduos são levados a um relaxamento corporal total e o corpo libera imediatamente a morfina natural, auxiliando, assim, no alívio da dor, de forma semelhante ao que acontece com o analgésico sintético ${ }^{25}$.

Embora a música esteja conectada com o sistema límbico, que é responsável pelas emoções, motivação e afeto, é escassa a produção científica sobre os efeitos da música nas alterações fisiológicas, psicológicas e emocionais, o que traz à tona a necessidade de mais investigações no campo da saúde ${ }^{26}$, sobretudo voltadas para crianças e adolescentes que se encontram com doenças ameaçadoras da vida. Integrar a análise de biomarcadores séricos com medidas psicológicas na assistência ao paciente vem se tornando a cada dia mais emergente no contexto da saúde, adoecimento e do cuidado personalizado ${ }^{27}$.

Em estudo, a presença de palhaços hospitalares durante a realização de procedimentos em crianças e adolescentes mostrou-se benéfica e auxiliou a melhora do estado psicológico desses, quando comparados com os que receberam apenas cuidados padrões. No estudo, recomendou-se a avaliação do impacto dos palhaços hospitalares e sua correlação com dados clínicos e biomarcadores ${ }^{28}$.

Outros autores destacam a necessidade da realização de novos estudos que avaliem os biomarcadores comuns no câncer de modo a auxiliar na utilização de intervenções que contribuam para o tratamento dos sintomas psiconeurológicos ${ }^{29}$.

Estudo com objetivo de avaliar o efeito da musicoterapia sobre o estresse de dependentes químicos verificou que após a intervenção terapêutica ocorreu a redução dos níveis de cortisol salivar, marcador bioquímico do estresse, reduzindo assim o estresse de dependentes químicos, mostrando desse modo os benefícios da musicoterapia na assistência ${ }^{30}$.
A música, como recurso terapêutico, destaca-se entre as práticas integrativas e complementares por promover alterações de origem biopsicossocial, auxiliando no tratamento dos pacientes e na melhoria da qualidade de vida. Quando utilizada em pacientes com doenças ameaçadoras da vida, promove a melhoria da comunicação e do relacionamento entre doente e família ${ }^{31}$.

Desse modo, é possível perceber a partir dos desenhos e depoimentos, os efeitos da musicoterapia durante o processo de hospitalização de crianças e adolescentes com câncer. Tendo em vista que este recurso pôde transformar aspectos negativos relacionados à situação vivenciada por aspectos positivos, auxiliando os participantes da pesquisa a esquecerem o processo de adoecimento e hospitalização e lutarem pela vida, a possuírem esperança de melhora do quadro, e no alívio da dor, contribuindo para a melhoria da qualidade de vida destes, mesmo diante da situação ameaçadora da vida e do processo de hospitalização.

\section{CONSIDERAÇÕES FINAIS E IMPLICAÇÕES PARA A PRÁTICA}

Constatou-se que as crianças e adolescentes, antes de receberem a musicoterapia, expressaram sentimentos de tristeza, medo e saudade relacionados ao rompimento do vínculo familiar no cenário domiciliar durante o processo de hospitalização.

Por sua vez, após receberem a musicoterapia, elas expressaram as sensações de prazer e bem-estar que essa vivência pôde Ihes proporcionar, permitindo-Ihes a expressão dos sentimentos mais profundos.

Percebeu-se que a musicoterapia possibilitou o resgate de lembranças positivas e a esperança em viver melhor; propiciou um espaço para a comunicação de anseios, medos e esperanças; e promoveu o alívio da dor. Tudo isso pode conferir uma melhor qualidade de vida às crianças e adolescentes com câncer durante a assistência paliativista.

Atribuem-se às limitações do estudo a não realização da pesquisa com crianças menores de oito anos acometidas com câncer em cuidados paliativos; com amostra aleatória; a não realização da quantificação dos benefícios da utilização da musicoterapia no adoecimento do público infantojuvenil; a não padronização do tipo de música selecionada aos participantes; e o reduzido quantitativo de participantes. Entretanto, salienta-se a qualidade e a riqueza do material empírico do presente estudo, não prejudicando as evidências da pesquisa.

Este estudo traz contribuições para a prática, uma vez que investiga a percepção de crianças e adolescentes com câncer sobre um recurso que pode ser utilizado em diversas instituições de saúde para beneficiar pacientes que se encontram diante de situações consideradas difíceis, principalmente dos que se encontram com uma doença que ameaça a vida e que também necessitam de terapias não farmacológicas para o alívio da dor e de outros sintomas que possam vir a ter. 


\section{CONTRIBUIÇÃO DOS AUTORES}

Desenho do estudo. Julia Helena Machado Franco. Carla Braz Evangelista.

Coleta ou produção dos dados. Julia Helena Machado Franco.

Análise de dados. Julia Helena Machado Franco. Carla Braz Evangelista. Mariana de Sousa Dantas Rodrigues. Ronny Anderson de Oliveira Cruz. Indaya Machado Freire Franco. Mayara Limeira Freire.

Interpretação dos resultados. Julia Helena Machado Franco. Carla Braz Evangelista. Mariana de Sousa Dantas Rodrigues. Ronny Anderson de Oliveira Cruz. Indaya Machado Freire Franco. Mayara Limeira Freire.

Redação e revisão crítica do manuscrito. Julia Helena Machado Franco. Carla Braz Evangelista. Mariana de Sousa Dantas Rodrigues. Ronny Anderson de Oliveira Cruz. Indaya Machado Freire Franco. Mayara Limeira Freire.

Aprovação da versão final do artigo. Julia Helena Machado Franco.Carla Braz Evangelista.Mariana de Sousa Dantas Rodrigues.Ronny Anderson de Oliveira Cruz. Indaya Machado Freire Franco. Mayara Limeira Freire.

Responsabilidade por todos os aspectos do conteúdo e a integridade do artigo publicado. Julia Helena Machado Franco. Carla Braz Evangelista.Mariana de Sousa Dantas Rodrigues. Ronny Anderson de Oliveira Cruz. Indaya Machado Freire Franco. Mayara Limeira Freire.

\section{EDITOR ASSOCIADO}

Eliane Tatsch Neves (D)

\section{EDITOR CIENTÍFICO}

Ivone Evangelista Cabral (D)

\section{REFERÊNCIAS}

1. World Health Organization. WHO definition of palliative care [Internet]. Geneva: WHO; 2018 [citado 2018 out 5]. Disponível em: https://www. who.int/cancer/palliative/definition/en/

2. Iglesias SBO, Zollner ACR, Constantino CF. Cuidados paliativos pediátricos. Resid Pediatr. 2016;6(Supl. 1):46-54. http://dx.doi. org/10.25060/residpediatr-2016.v6s1-10.

3. World Health Organization. Childhood câncer [Internet]. Geneva: WHO; 2021 [citado 2021 abr 1]. Disponível em: https://www.who.int/news-room/ fact-sheets/detail/cancer-in-children

4. França JRFS, Silva EC, Machado KOA, Oliveira TC, Silva MFOC, Freire MEM. Vivência de crianças com câncer sob assistência paliativa em uma casa de apoio. REME rev min enferm. 2017;21:e-1065. http:// dx.doi.org/10.5935/1415-2762.20170075.

5. Sousa ADRSE, Silva LFD, Paiva ED. Nursing interventions in palliative care in Pediatric Oncology: an integrative review. Rev Bras Enferm. 2019;72(2):531-54. http://dx.doi.org/10.1590/0034-7167-2018-0121. PMid:31017219.

6. Nunes MDR, Bomfim E, Olson K, Lopes-Junior LC, Silva-Rodrigues FM, Garcia de Lima RA et al. Interventions minimizing fatigue in children/ adolescents with cancer: An integrative review. J Child Health Care. 2018;22(2):186-204. http://dx.doi.org/10.1177/1367493517752498. PMid:29361838.
7. Lopes-Júnior LC, Bomfim EO, Nascimento LC, Nunes MDR, Pereirada-Silva G, Lima RAG. Non-pharmacological interventions to manage fatigue and psychological stress in children and adolescents with cancer: an integrative review. Eur J Cancer Care (Engl). 2016;25(6):921-35. http://dx.doi.org/10.1111/ecc.12381. PMid:26374619.

8. Lopes-Junior LC, Silveira DSC, Olson K, Bomfim EO, Veronez LC, Santos JC et al. Clown intervention on psychological stress and fatigue in pediatric patients with Cancer Undergoing Chemotherapy. Cancer Nurs. 2020;43(4):290-9. http://dx.doi.org/10.1097/NCC.0000000000000690. PMid:30801267.

9. Xavier WS, Pacheco ST, Silva LF, Nascimento LC, Lopes-Júnior LC Araújo $\mathrm{BB}$, et al. Intervenções não farmacológicas na melhoria da qualidade de vida de crianças/adolescentes oncológicos. Acta pau enferm. 2020;33:e-APE-20190022. http://dx.doi.org/10.37689/actaape/2020ar0022.

10. Lopes-Júnior LC, Rosa GS, Pessanha RM, Schuab SIPC, Nunes $\mathrm{KZ}$, Amorim MHC. Efficacy of the complementary therapies in the management of cancer pain in palliative care: a systematic review. Rev Lat Am Enfermagem. 2020;28:e3377. http://dx.doi.org/10.1590/15188345.4213.3377. PMid:33027406.

11. Bradt J, Dileo C, Magill L, Teague A. Music interventions for improving psychological and physical outcomes in cancer patients. Cochrane Database Syst Rev. 2016;(8):CD006911. http://dx.doi.org/10.1002/14651858. CD006911.pub3. PMid:27524661.

12. Tong A, Sainsbury P, Craig J. Consolidated criteria for reporting qualitative research (COREQ): a 32-item checklist for interviews and focus groups. Int J Qual Health Care. 2007;19(6):349-57. http://dx.doi.org/10.1093/ intqhc/mzm042. PMid:17872937.

13. França JR. Cuidados Paliativos: relação dialógica entre a enfermeira e a criança com cancer [tese]. João Pessoa: Programa de Pós-Graduação em Enfermagem, Centro de Ciências da Saúde, Universidade Federal da Paraíba; 2014.

14. Costa TS, Morais AC. A hospitalização infantil: vivência de crianças a partir de representações gráficas. Rev enferm UFPE on line. 2017;11(supl. 1):358-67. https://doi.org/10.5205/reuol.7995-699314-SM.1101sup201715.

15. Arcuri IG. O desenho-estória como linguagem - arte como processo psicodiagnóstico. Revista de Arteterapia da AATESP. [Internet]. 2013 [citado $2021 \mathrm{abr}$ 1];4(1):13-33. Disponível em: https://www.aatesp.com. br/resources/files/downloads/revista_v4_n1.pdf

16. Bardin L. Análise de conteúdo. Lisboa: Edições 70; 2016.

17. Resolução $n^{\circ}$ 466, de 2012 de dezembro de 2012 (BR). Publicada resolução 466 do CNS que trata de pesquisas em seres humanos e atualiza a resolução 196. Diário Oficial da União de Saúde, Brasília (DF), 2013.

18. Silveira KA, Paula KMP, Enumo SRF. Stress related to pediatric hospitalization and possible interventions: an analysis of the brazilian literature. Trends Psychol. 2019 jun;27(2):443-58. http://dx.doi.org/10.9788/tp2019.2-11.

19. Rodgers $\mathrm{C}$, Hooke MC, Ward J, Linder LA. Symptom clusters in children and adolescents with cancer. Semin Oncol Nurs. 2016;32(4):394-404. http://dx.doi.org/10.1016/j.soncn.2016.08.005. PMid:27776834.

20. Nóia TC, Sant'Ana RSE, Santos ADSD, Oliveira SC, Bastos Veras SMC Lopes-Júnior LC. Coping with the diagnosis and hospitalization of a child with childhood cancer. Invest Educ Enferm. 2015 dez;33(3):46572. http://dx.doi.org/10.17533/udea.iee.v33n3a10. PMid:28569954.

21. Depianti JRB, Melo L L, Ribeiro CA. Brincando para continuar a ser criança e libertar-se do confinamento da hospitalização em precaução. Esc Anna Nery Rev Enferm. 2018;22(2):e20170313. http://dx.doi. org/10.1590/2177-9465-ean-2017-0313.

22. da Silva MCM, Lopes LC Jr, Nascimento LC, Lima RAG. Fadiga em crianças e adolescentes com câncer sob a perspectiva dos profissionais de saúde. Rev Lat Am Enfermagem. 2016;24:e2784. http://dx.doi. org/10.1590/1518-8345.1159.2784.

23. Lesinskiene S, Lesinskaite A, Sambaras R, Karaliene V. Survey of drawing and art activities of preschoolers: attitudes and experiences of parentes. Health Education and Care. 2018;3(1):1-4. http://dx.doi. org/10.15761/HEC.1000134.

24. Andrade $\mathrm{H}$ Jr. Eficácia terapêutica da música: um olhar transdisciplinar de saúde para equipes, pacientes e acompanhantes. Rev enferm UERJ. 2018;26:e29155. http://dx.doi.org/10.12957/reuerj.2018.29155. 
25. Nemes MC, Souza LMFOL. Musicoterapia receptiva no tratamento da dor crônica. Revista InCantare. [Internet]. 2018 [citado 2021 abr 1];9(1):47-66. Disponível em: http://periodicos.unespar.edu.br/index. php/incantare/article/view/2394

26. Campos L, Nakasu M. Efeitos da utilização da música no ambiente hospitalar: revisão sistemática. Revista Sonora. [Internet]. 2016 [citado 2021 abr 1];6(11):1-11. Disponível em: https://www.publionline.iar. unicamp.br/index.php/sonora/article/view/686

27. Amorim MHC, Lopes LC Jr. Psiconeuroimunologia e a pesquisa em Enfermagem: descoberta, mudanças de paradigma e inovações metodológicas. Acta paul enferm. 2021;34:e-EDT1. http://dx.doi. org/10.37689/acta-ape/2021edt1.

28. Lopes-Júnior LC, Bomfim E, Olson K, Neves ET, Silveira DSC, Nunes MDR et al. Effectiveness of hospital clowns for symptom management in paediatrics: systematic review of randomised and non-randomised controlled trials. BMJ.2020;371:m4290. http://dx.doi.org/10.1136/bmj. m4290. PMid:33328164.

29. Lopes-Júnior LC, Olson K, de Omena Bomfim E, Pereira-da-Silva G, Nascimento LC, de Lima RA. Translational research and symptom management in oncology nursing. Br J Nurs. 2016;25(10):S12S14, S16 passim. http://dx.doi.org/10.12968/bjon.2016.25.10.S12. PMid:27231745.

30. Taets GGC, Jomar RT, Abreu AMM, Capella MAM. Effect of music therapy on stress in chemically dependent people: a quasi-experimental study. Rev Lat Am Enfermagem. 2019 jan 17;27:e3115. http://dx.doi. org/10.1590/1518-8345.2456.3115. PMid:30698217.

31. Oliveira MF, Oselame GB, Neves EB, Oliveira EM. Musicoterapia como ferramenta terapêutica no setor da saúde: uma revisão sistemática. Rev Univ Vale Rio Verde. 2014;12(2):871-8. http://dx.doi.org/10.5892/ ruvrd.v12i2.1739. 